University of Nebraska - Lincoln

DigitalCommons@University of Nebraska - Lincoln

2006

\title{
The Dielectric Behavior of Polycrystalline Ferroelectric Films with Fiber Textures
}

\author{
Quangen Du \\ Department of Mechanical Engineering, University of Washington, Box 352600, Seattle, WA \\ Jiangyu Li \\ University of Washington, jjli@uw.edu \\ William Nothwang \\ Weapons and Materials Research Directorate, US Army Research Laboratory, USA \\ Melanie W. Cole \\ Weapons and Materials Research Directorate, US Army Research Laboratory, USA
}

Follow this and additional works at: https://digitalcommons.unl.edu/usarmyresearch

Part of the Operations Research, Systems Engineering and Industrial Engineering Commons

Du, Quangen; Li, Jiangyu; Nothwang, William; and Cole, Melanie W., "The Dielectric Behavior of Polycrystalline Ferroelectric Films with Fiber Textures" (2006). US Army Research. 32.

https://digitalcommons.unl.edu/usarmyresearch/32

This Article is brought to you for free and open access by the U.S. Department of Defense at DigitalCommons@University of Nebraska - Lincoln. It has been accepted for inclusion in US Army Research by an authorized administrator of DigitalCommons@University of Nebraska - Lincoln. 


\title{
The dielectric behavior of polycrystalline ferroelectric films with fiber textures
}

\author{
Quangen Du ${ }^{\text {a }}$, JiangYu Li ${ }^{\text {a,* }}$, William Nothwang ${ }^{\mathrm{b}}$, Melanie W. Cole ${ }^{\mathrm{b}}$ \\ ${ }^{a}$ Department of Mechanical Engineering, University of Washington, Box 352600, Seattle, WA 98195 2600, USA \\ ${ }^{\mathrm{b}}$ Weapons and Materials Research Directorate, US Army Research Laboratory, USA
}

Received 12 November 2005; received in revised form 30 January 2006; accepted 30 January 2006

Available online 3 April 2006

\begin{abstract}
We consider the effects of crystallographic symmetry, texture, and internal strain on the dielectric properties of polycrystalline ferroelectric thin films with fiber textures, including spontaneous polarization, coercivity, dielectric constant, dielectric tunability, and strain-induced phase transition, by combining Landau-Ginzburg-Devonshire phenomenological theory for ferroelectrics with the micromechanics theory for polycrystalline thin films. Cubic and tetragonal $\mathrm{Ba}_{x} \mathrm{Sr}_{1-x} \mathrm{TiO}_{3}$ (BST) films with [0 01 1] fiber texture, and cubic and rhombohedral BST films with [ $\left.\begin{array}{lll}1 & 1 & 1\end{array}\right]$ texture are considered. The results show that for $\left[\begin{array}{lll}0 & 0 & 1\end{array}\right]$-textured film compressive strain enhances the spontaneous polarization, coercivity, and tunability while it decreases the dielectric constant; for [1 11 ]-textured film the effects are the opposite. It is also noted that [ 1111$]$-textured films are less sensitive to internal strain than [ 001 1]-textured films. Good agreement with experimental data is also observed.
\end{abstract}

(C) 2006 Acta Materialia Inc. Published by Elsevier Ltd. All rights reserved.

Keywords: Ferroelectric film; Dielectric behavior; Fiber texture; Tunability

\section{Introduction}

Over the last decade ferroelectric thin films have attracted considerable attention because of their unique ferroelectric, dielectric, and piezoelectric properties, which are promising for microelectronic and micromechanical applications in sensors, actuators, transducers, capacitors, or memory cells [1]. The functional properties of ferroelectric thin films are usually significantly different from those of their bulk counterparts, due to the intrinsic or extrinsic size effects associated with film thickness and the influence of substrate [2-4]. In particular, it was found that the dielectric constant of heteroepitaxial $\mathrm{Ba}_{x} \mathrm{Sr}_{1-x} \mathrm{TiO}_{3}$ (BST) film is smaller than the bulk value and decreases rapidly for films less than $100 \mathrm{~nm}$ thick [5], which is attributed to the influence of internal stress induced by the substrate constraint [6]. The dielectric properties of BST are impor-

\footnotetext{
* Corresponding author. Tel.: +1 206685 2429; fax: +1 2066858047.

E-mail address: jjli@u.washington.edu (J. Li).
}

tant for potential applications in frequency-agile microwave devices due to the large dielectric tunability $[7,8]$.

Most theoretical models of ferroelectric thin films are based on Landau-Ginzburg-Devonshire phenomenological theory [9-11]. Under this framework, the effects of substrate constraint, internal stress, and film thickness have been successfully analyzed, and phase diagrams have been constructed $[5,6]$. Most of the previous studies focused on epitaxial single-crystalline thin films, without addressing the important influence of intergranular constraint [12] in polycrystalline films. This paper is intended to address this deficiency. In particular, we seek to combine LandauGinzburg-Devonshire phenomenological theory for ferroelectrics with the micromechanics theory for polycrystalline thin films, which allows us to study the overall behavior of a polycrystalline ferroelectric thin film in terms of its microstructure, especially the texture and internal strain. We are especially interested in films with fiber textures, which can be analyzed rigorously without approximations regarding the distribution of electromechanical field in the film 
$[13,14]$. In fact, such fiber textures are commonly observed in thin-film deposition. For example, highly [0 011$]$-textured BST films have been grown by liquid-source metalorganic chemical vapor deposition [15], and [1 11 ]-oriented $\mathrm{Pb}(\mathrm{Zr}, \mathrm{Ti}) \mathrm{O}_{3}$ (PZT) films have been deposited using they sol-gel technique [16], with grains in both films being columnar.

The importance of internal stress and strain in polycrystalline thin films is widely recognized, which has a significant influence on the dielectric behavior of BST thin films through the electrostrictive effect and phase transitions $[6,10,11]$. Generally speaking, the internal stress and strain can be experimentally adjusted by varying the film thickness [5,6], by selecting different substrates [17], by changing the deposition conditions, and by post-annealing processes [18]. Recently, Morito and Suzuki [21] have reported a detailed experimental investigation on the effects of residue strain on the microstructure and dielectric properties of sputter-deposited polycrystalline BST thin films. In addition, the effects of strain gradient [19] and nonequally biaxial misfit strain [20] on the dielectric properties of epitaxial ferroelectric thin films have also been reported. However, such effects, despite their importance, have rarely been studied theoretically other than in epitaxial single-crystalline thin films.

The paper is organized as follows. The phenomenological model for ferroelectrics is introduced first in Section 2, where the nonlinear electromechanical constitutive equations are derived from their thermodynamic potential, with special attention being paid to films consisting of crystalline grains of cubic symmetry. Ferroelectric films with fiber textures are considered in Section 3, where cubic and tetragonal films with [0 011$]$ texture and cubic and rhombohedral films with [111] texture are studied in detail after general considerations. Numerical results and discussions are then presented in Section 4.

\section{Phenomenological model of ferroelectrics}

\subsection{Energetics and constitutive equations}

We consider a ferroelectric thin film $\Omega$ sandwiched between a top electrode and a substrate, with the potential energy given by

$F(\boldsymbol{\sigma}, \mathbf{P})=\int_{\Omega}\left(W(\boldsymbol{\sigma}, \mathbf{P})-\mathbf{E}_{0} \cdot \mathbf{P}+\boldsymbol{\varepsilon}^{0} \cdot \boldsymbol{\sigma}\right) \mathrm{d} \mathbf{x}+\frac{\varepsilon_{0}}{2} \int_{\mathbb{R}^{3}}|\nabla \phi|^{2} \mathrm{~d} \mathbf{x}$

where the first term is the 'stored energy density' of the ferroelectric which depends on the state variables, stress $\sigma$ and polarization $\mathbf{P}$. At any given $\mathbf{x}, W(\boldsymbol{\sigma}, \mathbf{P})$ encodes the information that the ferroelectric prefers certain states of stress and polarization, as we will elaborate later. In particular, it has a multi-well structure, leading to 'variants' of different transformation strain and polarization. The second term is the potential associated with the applied electric field $\mathbf{E}_{0}$ which tends to align the polarization towards $\mathbf{E}_{0}$, and the third term is the energy associated with the applied mechanical constraint, where $\varepsilon^{0}$ is the strain consistent with the mechanical constraint imposed by the substrate, the misfit strain; it can also be the residue strain induced during thin-film deposition. The final term is the depolarization energy due to the electric field generated by the polarization distribution in the ferroelectric, where $\varepsilon_{0}=8.85 \times$ $10^{-12} \mathrm{C}^{2} / \mathrm{N} \mathrm{m}^{2}$ is the permittivity of free space. The electric potential $\phi$ is obtained by solving Maxwell's equation

$\nabla \cdot\left(-\varepsilon_{0} \nabla \phi+\mathbf{P} \chi_{\Omega}\right)=\nabla \cdot\left(\varepsilon_{0} \mathbf{E}^{\prime}+\mathbf{P} \chi_{\Omega}\right)=\rho_{\mathrm{f}}$

subject to appropriate boundary conditions, where $\mathbf{E}^{\prime}=-\nabla \phi$ is the electric field induced by the polarization distribution, $\chi_{\Omega}$ is the characteristic function of $\Omega$ and $\rho_{\mathrm{f}}$ is the free charge density. We ignore the interface energy that penalizes the polarization gradient, which is appropriate if the size of the ferroelectric is much larger than the correlation length of polarization [22]. This type of potential energy of a ferroelectric is derived from the one established by Shu and Bhattacharya [23], who used strain rather than stress as an independent variable.

In general, we can expand $W$ as a polynomial of $\sigma$ up to the second order and $\mathbf{P}$ up to the fourth order $[9,10]$, resulting in

$$
\begin{aligned}
W(\boldsymbol{\sigma}, \mathbf{P})= & \frac{1}{2} \mathbf{P} \cdot \boldsymbol{\beta P}+\frac{1}{2}(\mathbf{P} \otimes \mathbf{P}) \cdot \mathbf{a}(\mathbf{P} \otimes \mathbf{P})-\frac{1}{2} \boldsymbol{\sigma} \cdot \mathbf{s} \boldsymbol{\sigma} \\
& -(\mathbf{P} \otimes \mathbf{P}) \cdot \mathbf{Q} \boldsymbol{\sigma}
\end{aligned}
$$

where $\boldsymbol{\beta}$ is the second-rank dielectric tensor, $\mathbf{a}$ is the fourthrank dielectric tensor, $\mathbf{s}$ is the fourth-rank elastic compliance tensor, and $\mathbf{Q}$ is the fourth-rank electrostrictive tensor. The stress and polarization adopted by a ferroelectric in an equilibrium state will then maximize and minimize the potential energy, respectively, leading to

$\frac{\delta F}{\delta \boldsymbol{\sigma}}=\mathbf{0}, \quad \frac{\delta F}{\delta \mathbf{P}}=\mathbf{0}$

which results in the following constitutive equations:

$$
\begin{aligned}
\boldsymbol{\varepsilon}^{0}= & -\frac{\partial W}{\partial \boldsymbol{\sigma}}=\mathbf{s} \boldsymbol{\sigma}+\mathbf{Q}(\mathbf{P} \otimes \mathbf{P}), \\
\mathbf{E}= & \mathbf{E}_{0}+\mathbf{E}^{\prime}=\frac{\partial W}{\partial \mathbf{P}}=\boldsymbol{\beta P}+\frac{\partial(\mathbf{P} \otimes \mathbf{P})}{\partial \mathbf{P}} \cdot \mathbf{a}(\mathbf{P} \otimes \mathbf{P}) \\
& -\frac{\partial(\mathbf{P} \otimes \mathbf{P})}{\partial \mathbf{P}} \cdot \mathbf{Q} \boldsymbol{\sigma}
\end{aligned}
$$

where $\mathbf{E}$ is the total electric field in the thin film, consisting of the external electric field applied by the electrodes and the internal depolarization field induced by the polarization distribution. In the constitutive equation, the stress and polarization are nonlinearly coupled, where the electrostrictive stain is dependent on the polarization quadratically, and the electric field is dependent on stress linearly, both through the electrostrictive tensor $\mathbf{Q}$. High-order dielectric response is considered by the fourth-rank dielectric tensor a. We point out that instead of working with the potential energy (Eq. (3)) directly, which is common for the study of single-crystalline thin films, coupled constitutive 
equations (Eqs. (5)) have to be used to study ferroelectric polycrystalline films, which generally have heterogeneous electromechanical field distribution that can be more conveniently determined from the constitutive equations.

\subsection{Ferroelectrics with cubic symmetry}

To be specific, let us consider a ferroelectric possessing cubic symmetry at high temperature, with the constitutive moduli written in the following matrix forms [24]: where

$$
\mathbf{P}^{2}=\left(\begin{array}{c}
P_{1}^{2} \\
P_{2}^{2} \\
P_{3}^{2} \\
P_{2} P_{3} \\
P_{1} P_{3} \\
P_{1} P_{2}
\end{array}\right), \quad \hat{\mathbf{P}}=\left(\begin{array}{cccccc}
2 P_{1} & 0 & 0 & 0 & P_{3} & P_{2} \\
0 & 2 P_{2} & 0 & P_{3} & 0 & P_{1} \\
0 & 0 & 2 P_{3} & P_{2} & P_{1} & 0
\end{array}\right)
$$

$\boldsymbol{\beta}=\left(\begin{array}{ccc}\beta_{1} & 0 & 0 \\ 0 & \beta_{1} & 0 \\ 0 & 0 & \beta_{1}\end{array}\right), \quad \mathbf{a}=\left(\begin{array}{ccccccc}a_{11} & b & b & 0 & 0 & 0 \\ b & a_{11} & b & 0 & 0 & 0 \\ b & b & a_{11} & 0 & 0 & 0 \\ 0 & 0 & 0 & a_{12}-2 b & 0 & 0 \\ 0 & 0 & 0 & 0 & a_{12}-2 b & 0 \\ 0 & 0 & 0 & 0 & 0 & a_{12}-2 b\end{array}\right)$,
$\mathbf{s}=\left(\begin{array}{ccccccccc}s_{11} & s_{12} & s_{12} & 0 & 0 & 0 \\ s_{12} & s_{11} & s_{12} & 0 & 0 & 0 \\ s_{12} & s_{12} & s_{11} & 0 & 0 & 0 \\ 0 & 0 & 0 & s_{44} & 0 & 0 \\ 0 & 0 & 0 & 0 & s_{44} & 0 \\ 0 & 0 & 0 & 0 & 0 & s_{44}\end{array}\right), \quad \mathbf{Q}=\left(\begin{array}{cccccc}Q_{11} & Q_{12} & Q_{12} & 0 & 0 & 0 \\ Q_{12} & Q_{11} & Q_{12} & 0 & 0 & 0 \\ Q_{12} & Q_{12} & Q_{11} & 0 & 0 & 0 \\ 0 & 0 & 0 & Q_{44} & 0 & 0 \\ 0 & 0 & 0 & 0 & Q_{44} & 0 \\ 0 & 0 & 0 & 0 & 0 & Q_{44}\end{array}\right)$

The temperature-dependent dielectric stiffness $\beta_{1}$ is given by the well-known Curie-Weiss law

$\beta_{1}=\frac{T-T_{0}}{2 \varepsilon_{0} C}$

where $T_{0}$ and $C$ are the Curie-Weiss temperature and constant, respectively. We adopt the usual conversion rule between tensor and matrix representations of $\mathbf{s}, \boldsymbol{\sigma}$, and $\boldsymbol{\varepsilon}$ [24]; the conversion rules for fourth-rank tensor $\mathbf{Q}$ and $\mathbf{a}$ are similar to $\mathbf{s}$. These conversions are necessary for tensor rotation when grains at different orientations are considered. With these constitutive moduli given in matrix notation, the stored energy density can then be written as

$$
\begin{aligned}
W= & \frac{1}{2} \beta_{1}\left(P_{1}^{2}+P_{2}^{2}+P_{3}^{2}\right)+\frac{1}{2} a_{11}\left(P_{1}^{4}+P_{2}^{4}+P_{3}^{4}\right) \\
& +\frac{1}{2} a_{12}\left(P_{1}^{2} P_{2}^{2}+P_{1}^{2} P_{3}^{2}+P_{2}^{2} P_{3}^{2}\right)-\frac{1}{2} s_{11}\left(\sigma_{1}^{2}+\sigma_{2}^{2}+\sigma_{3}^{2}\right) \\
& -s_{12}\left(\sigma_{1} \sigma_{2}+\sigma_{1} \sigma_{3}+\sigma_{2} \sigma_{3}\right)-\frac{1}{2} s_{44}\left(\sigma_{4}^{2}+\sigma_{5}^{2}+\sigma_{6}^{2}\right) \\
& -Q_{11}\left(\sigma_{1} P_{1}^{2}+\sigma_{2} P_{2}^{2}+\sigma_{3} P_{3}^{2}\right)-Q_{44}\left(\sigma_{4} P_{2} P_{3}+\sigma_{5} P_{3} P_{1}\right. \\
& \left.+\sigma_{6} P_{1} P_{2}\right)-Q_{12}\left[\sigma_{1}\left(P_{2}^{2}+P_{3}^{2}\right)+\sigma_{2}\left(P_{1}^{2}+P_{3}^{3}\right)+\sigma_{3}\left(P_{1}^{2}+P_{2}^{2}\right)\right]
\end{aligned}
$$

resulting in the following constitutive equations in matrix form:

$\boldsymbol{\varepsilon}=\mathbf{s} \boldsymbol{\sigma}+\mathbf{Q P}^{2}, \quad \mathbf{E}=\boldsymbol{\beta P}+\hat{\mathbf{P}} \mathbf{a} \mathbf{P}^{2}-\hat{\mathbf{P}} \mathbf{Q} \boldsymbol{\sigma}$
This is the constitutive equation for a ferroelectric crystal with cubic symmetry, which serves as the input in our study of polycrystalline ferroelectric thin films.

\section{Ferroelectric films with fiber texture}

\subsection{General considerations}

We now consider a ferroelectric polycrystalline film consisting of numerous grains of different orientations. For each of the grains, the constitutive behavior is assumed known, given by Eq. (7) in their respective local coordinate system $\mathbf{X}$ fixed on individual grains, with $X_{i}$ aligned along certain crystallographic directions. What we are interested in is the overall response of the polycrystalline film that has to be given in a global coordinate, $\mathbf{x}$, which is fixed on the film and thus is common for all grains. In addition, we assume that the film possesses fiber texture, i.e., each grain is columnar and has its $X_{3}$ aligned along the normal direction of the film, the $x_{3}$ axis of the global coordinate. Within the $x_{1}-x_{2}$ plane, the texture is arbitrary. As a result, the orientation of each grain can be described by a single variable $\theta$, which is the angle between $X_{1}$ of the grain and $x_{1}$ of the film (see Fig. 1). Such fiber texture is not only convenient for mathematical analysis, but also quite commonly observed in experiments $[15,16]$. 


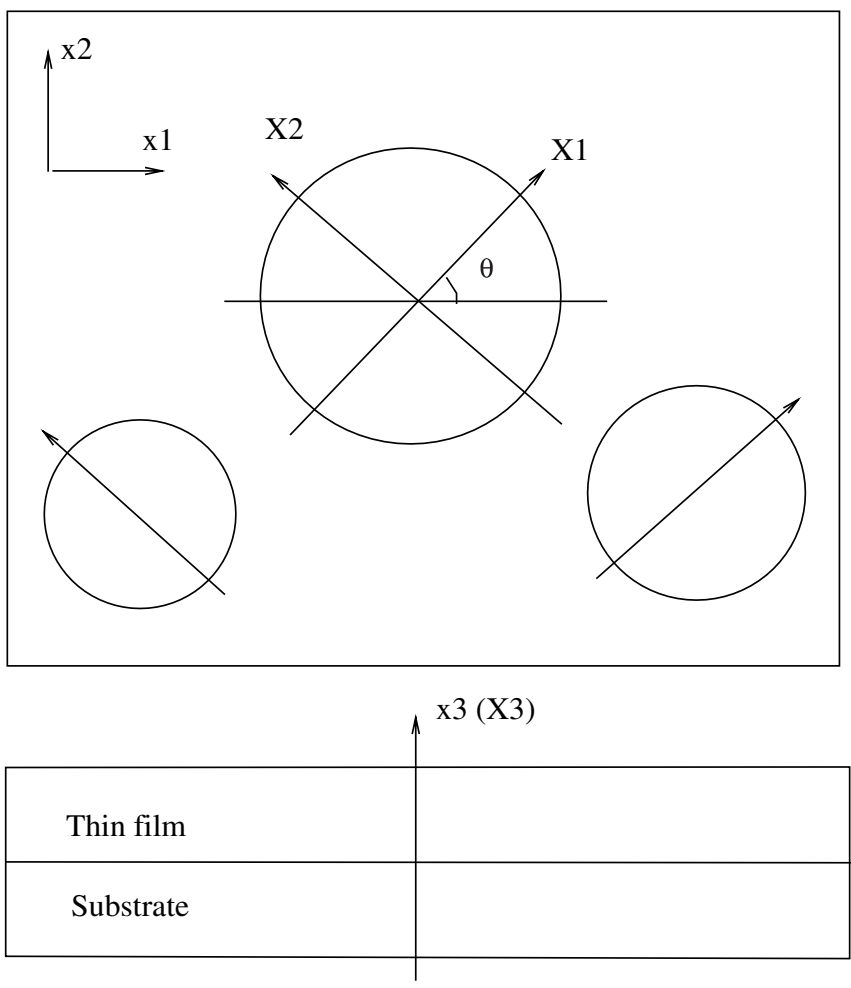

Fig. 1. Schematic of a polycrystalline ferroelectric thin film with fiber texture.

The thin-film microstructure is different from that of bulk materials in a couple of aspects. First, it is strongly constrained by the substrate, which imposes a biaxial misfit strain $\varepsilon^{0}$ along the $x_{1}$ and $x_{2}$ directions. Second, each grain in the thin film is strongly constrained by its neighboring grains, and thus cannot deform freely. As a result, the electromechanical behavior of such a fiber-textured thin film is markedly different from that of bulk materials. In order to determine the electromechanical response of the film subjected to an applied electric field $E^{0}$ along $x_{3}$, the distribution of electromechanical field in each grain has to be determined first and then averaged. This is not a trivial problem in general, but can be solved exactly for certain fiber textures. Here we summarize our solution strategy. First, we decompose the thin film into individual grains subject to the biaxial constraint from the substrate, but free of constraint from its neighbors. As a result, its electromechanical field can be determined. We then notice that the electromechanical field in each of the grains will not depend on its orientation $\theta$ under certain crystallographic symmetries and fiber textures, which allows us to reassemble individual grains into a thin film without any disturbance. Finally, we average the electromechanical field over all grains to yield the overall response of the thin film under the applied electric field, from which the electromechanical properties, particularly the dielectric properties in which we are interested, can be determined. We follow this procedure in the following analysis.
For a ferroelectric film subjected to an applied electric field $E^{0}$ along the $x_{3}$ axis and biaxial misfit strain $\varepsilon^{0}$ along the $x_{1}$ and $x_{2}$ directions, with the top surface traction free, some components of average electric field, strain, and stress in the film can be determined from the boundary conditions as

$$
\begin{aligned}
& \langle\mathbf{E}\rangle=\left(0,0, E^{0}\right), \quad\left\langle\varepsilon_{1}\right\rangle=\left\langle\varepsilon_{2}\right\rangle=\varepsilon^{0},\left\langle\varepsilon_{6}\right\rangle=0, \\
& \left\langle\sigma_{3}\right\rangle=\left\langle\sigma_{4}\right\rangle=\left\langle\sigma_{5}\right\rangle=0
\end{aligned}
$$

where \langle\rangle is used to denote orientational averaging over all grains. Within the thin film, the electromechanical field could vary from grain to grain due to the heterogeneity. They can be determined, however, from the above-mentioned imaginary cut-paste experiment. First, we disassemble the film into individual grains, and rotate the electromechanical loading applied at the boundary of thin film from the global coordinate to local coordinates of each individual grain, so that for a grain at $\theta$, we have

$\boldsymbol{\sigma}(\theta)=\left\{\sigma_{1}(\theta), \sigma_{2}(\theta), 0,0,0, \sigma_{6}(\theta)\right\}^{\mathrm{T}}$,

$\boldsymbol{\varepsilon}(\theta)=\left\{\varepsilon^{0}, \varepsilon^{0}, \varepsilon_{3}(\theta), \varepsilon_{4}(\theta), \varepsilon_{5}(\theta), 0\right\}^{\mathrm{T}}, \quad \mathbf{E}(\theta)=\left\{0,0, E^{0}\right\}^{\mathrm{T}}$

where three components of stress and three components of strain are unknown and have to be determined. For a grain at orientation $\theta$, as a result, the constitutive equations in the local coordinates are given by:

$$
\begin{aligned}
& \boldsymbol{\varepsilon}(\theta)=\mathbf{s} \boldsymbol{\sigma}(\theta)+\mathbf{Q P}^{2}(\theta) \\
& \mathbf{E}=\boldsymbol{\beta P}(\theta)+\hat{\mathbf{P}}(\theta) \mathbf{a} \mathbf{P}^{2}(\theta)-\hat{\mathbf{P}}(\theta) \mathbf{Q} \boldsymbol{\sigma}(\theta)
\end{aligned}
$$

Since we will consider both [001] and [111] fiber textures, the local coordinates might not be aligned along the principal axes of cubic symmetry, and the local compliance moduli can in general be expressed as

$$
\mathbf{s}=\left(\begin{array}{cccccc}
s_{11} & s_{12} & s_{13} & 0 & s_{15} & 0 \\
s_{12} & s_{11} & s_{12} & 0 & s_{25} & 0 \\
s_{13} & s_{13} & s_{11} & 0 & 0 & 0 \\
0 & 0 & 0 & s_{44} & 0 & s_{46} \\
s_{15} & s_{25} & 0 & 0 & s_{44} & 0 \\
0 & 0 & 0 & s_{46} & 0 & s_{66}
\end{array}\right)
$$

for both [001] and [1 111 ] fiber textures, with $\mathbf{Q}$ and $\mathbf{a}$ having similar structures. In order to solve the equations to determine the unknown electromechanical field, we rearrange Eq. (9) as follows:

$$
\left(\begin{array}{l}
\mathbf{Y} \\
\mathbf{Z}
\end{array}\right)=\left(\begin{array}{cc}
\mathbf{V} & \mathbf{B} \\
\mathbf{B}^{\mathrm{T}} & \mathbf{N}
\end{array}\right)\left(\begin{array}{c}
\mathbf{F} \\
\mathbf{G}
\end{array}\right)+\left(\begin{array}{cc}
\mathbf{C} & \mathbf{D} \\
\mathbf{D}^{\mathrm{T}} & \mathbf{M}
\end{array}\right)\left(\begin{array}{l}
\mathbf{K} \\
\mathbf{L}
\end{array}\right)
$$


where

$$
\begin{aligned}
& \mathbf{Y}=\left(\begin{array}{c}
\varepsilon^{0} \\
\varepsilon^{0} \\
0
\end{array}\right), \quad \mathbf{Z}=\left(\begin{array}{l}
\varepsilon_{3}(\theta) \\
\varepsilon_{4}(\theta) \\
\varepsilon_{5}(\theta)
\end{array}\right), \quad \mathbf{F}=\left(\begin{array}{c}
\sigma_{1}(\theta) \\
\sigma_{2}(\theta) \\
\sigma_{6}(\theta)
\end{array}\right), \quad \mathbf{G}=\left(\begin{array}{l}
0 \\
0 \\
0
\end{array}\right), \\
& \mathbf{K}=\left(\begin{array}{c}
P_{1}^{2}(\theta) \\
P_{2}^{2}(\theta) \\
P_{1}(\theta) P_{2}(\theta)
\end{array}\right), \quad \mathbf{L}=\left(\begin{array}{c}
P_{3}^{2}(\theta) \\
P_{2}(\theta) P_{3}(\theta) \\
P_{3}(\theta) P_{1}(\theta)
\end{array}\right), \\
& \mathbf{V}=\left(\begin{array}{ccc}
s_{11} & s_{12} & 0 \\
s_{12} & s_{11} & 0 \\
0 & 0 & s_{66}
\end{array}\right), \quad \mathbf{B}=\left(\begin{array}{ccc}
s_{13} & 0 & s_{15} \\
s_{13} & 0 & s_{25} \\
0 & s_{46} & 0
\end{array}\right), \\
& \mathbf{N}=\left(\begin{array}{ccc}
s_{11} & 0 & 0 \\
0 & s_{44} & 0 \\
0 & 0 & s_{44}
\end{array}\right), \quad \mathbf{C}=\left(\begin{array}{ccc}
Q_{11} & Q_{12} & 0 \\
Q_{12} & Q_{11} & 0 \\
0 & 0 & Q_{66}
\end{array}\right), \\
& \mathbf{D}=\left(\begin{array}{ccc}
Q_{13} & 0 & Q_{15} \\
Q_{13} & 0 & Q_{25} \\
0 & Q_{46} & 0
\end{array}\right), \quad \mathbf{M}=\left(\begin{array}{ccc}
Q_{11} & 0 & 0 \\
0 & Q_{44} & 0 \\
0 & 0 & Q_{44}
\end{array}\right)
\end{aligned}
$$

Notice that $\mathbf{Y}$ and $\mathbf{G}$ are known strain and stress components, while $\mathbf{Z}$ and $\mathbf{F}$ are unknown strain and stress components that need to be determined. Solving Eq. (11), we obtain:

$$
\begin{aligned}
\mathbf{F}= & \mathbf{V}^{-1}(\mathbf{Y}-\mathbf{B G}-\mathbf{C} \mathbf{K}-\mathbf{D} \mathbf{L}) \\
\mathbf{Z}= & \mathbf{B}^{\mathrm{T}} \mathbf{V}^{-1} \mathbf{Y}+\left(\mathbf{N}-\mathbf{B}^{\mathrm{T}} \mathbf{V}^{-1} \mathbf{B}\right) \mathbf{G} \\
& +\left(\mathbf{D}^{\mathrm{T}}-\mathbf{B}^{\mathrm{T}} \mathbf{V}^{-1} \mathbf{C}\right) \mathbf{K}+\left(\mathbf{M}-\mathbf{B}^{\mathrm{T}} \mathbf{V}^{-1} \mathbf{D}\right) \mathbf{L}
\end{aligned}
$$

in the local coordinates of the grain at orientation $\theta$. A similar technique has been applied to calculate the effective constitutive moduli of ferroelectric single crystals with engineered domain configurations [25-27]. We then rotate the solved electromechanical field in each grain from the local coordinates to global coordinates, and after the transformation it turns out that they do not depend on the orientation if the grain is either tetragonal with $[001]$ fiber texture or rhombohedral with [1 111 ] fiber texture, as we show in Sections 3.2 and 3.3. As a result, the electromechanical field in those thin films is uniform, which allows us to reassemble the grains into the thin film without any disturbance. This uniform electromechanical field is also the averaged electromechanical field of the thin film for which we are seeking. This completes the imaginary cut-paste experiment.

In order to calculate the stress and strain in the film, we still need to determine the polarization in individual grains under the applied electric field, which requires considering the specific symmetry and is pursued in Sections 3.2 and 3.3. We are particularly interested in the variation of dielectric constant as a function of the applied electric field, which can be determined from

$\chi_{33}\left(E^{0}\right)=\frac{\partial P_{3}}{\partial E^{0}}$

The dielectric tunability $\phi$, an important figure of merit for microwave applications, can then be derived from the var- iation of the dielectric constant with respect to the applied electric field [11]:

$\phi=1-\frac{\chi_{33}\left(E^{0}\right)}{\chi_{33}(0)}$

\subsection{Cubic and tetragonal phases with [001] fiber texture}

A ferroelectric crystal is in the tetragonal phase with spontaneous polarization at a temperature lower than the Curie temperature. For a polycrystalline film consisting of grains of tetragonal phase with their [001] directions aligned along the $x_{3}$-axis, the spontaneous polarization of a grain at orientation $\theta$ is given by

$P_{1}=P_{2}=0, \quad P_{3}(\theta)=0$

In contrast, at a temperature higher than the Curie temperature, the crystal is in the cubic phase with no spontaneous polarization:

$P_{1}=P_{2}=P_{3}=0$

which can be considered as a special case of the tetragonal phase. In either phase, we have the following relationship among the constitutive moduli derived from material symmetry:

$s_{12}=s_{13}, \quad s_{44}=s_{66}, \quad s_{15}=s_{25}=0, \quad s_{46}=0$

which allows us to determine the unknown stress and strain components in terms of polarization under local coordinate system by solving Eq. (12):

$\sigma_{1}(\theta)=\sigma_{2}(\theta)=\frac{\varepsilon^{0}-P_{3}^{2}(\theta) Q_{12}}{s_{11}+s_{12}}, \quad \sigma_{6}(\theta)=0$
$\varepsilon_{3}(\theta)=\frac{2 s_{12} \varepsilon^{0}\left[Q_{11}\left(s_{11}+s_{12}\right)-2 Q_{12} s_{12}\right] P_{3}^{2}(\theta)}{s_{11}+s_{12}}$,
$\varepsilon_{4}(\theta)=\varepsilon_{5}(\theta)=0$

Meanwhile, from Eq. (10) we obtain the equation for polarization of grain at orientation $\theta$ :

$\beta_{1} P_{3}(\theta)+2 a_{11} P_{3}^{3}(\theta)-2 Q_{12}\left[\sigma_{1}(\theta)+\sigma_{2}(\theta)\right] P_{3}(\theta)-E^{0}=0$

Substituting Eq. (15) into the above polarization equation, we obtain

$\left(\beta_{1}-\frac{4 Q_{12} \varepsilon^{0}}{s_{11}+s_{12}}\right) P_{3}(\theta)+2\left(a_{11}+\frac{2 Q_{12}^{2}}{s_{11}+s_{12}}\right) P_{3}^{3}(\theta)-E^{0}=0$

By introducing two nominal dielectric constants

$\beta_{1}^{*}=\beta_{1}-\frac{4 Q_{12} \varepsilon^{0}}{s_{11}+s_{12}}, \quad a_{1}^{*}=a_{11}+\frac{2 Q_{12}^{2}}{s_{11}+s_{12}}$

Eq. (17) can be rewritten as

$\beta_{1}^{*} P_{3}(\theta)+2 a_{11}^{*} P_{3}^{3}(\theta)-E^{0}=0$

and the cubic-tetragonal phase transition temperature can be determined from $\beta_{1}^{*}=0$ as 
$T_{\mathrm{c}}=T_{0}+\frac{8 C \varepsilon_{0} Q_{12}}{S_{11}+s_{12}} \varepsilon^{0}$

Clearly the polarization $P_{3}$ does not depend on $\theta$ in local coordinates, and neither do $\sigma_{1}, \sigma_{2}$, and $\varepsilon_{3}$. Since both electric field and polarization are uniaxial, and both stress and strain are equi-biaxial, the electromechanical field will remain unchanged after transforming back to global coordinates, suggesting that the electromechanical field due to the specified boundary condition is uniform in the thin film. This allows us to reassemble the grains into the film with- which also includes the cubic phase as a special case where the spontaneous polarization is zero. When the thin film has [111] fiber texture, with each grain having its [1111] direction along the $x_{3}$-axis, it is more convenient to choose the local coordinates $X_{1}, X_{2}$, and $X_{3}$ along [ $\left.11 \overline{2}\right]$, [ $\left[\begin{array}{ll}1 & 10\end{array}\right]$, and [111] directions, respectively. Under these local coordinates, the polarization for grain at orientation $\theta$ is

$\mathbf{P}^{\prime}=[0,0, \sqrt{3} P(\theta)]$

and the electromechanical constitutive moduli are:

$\mathbf{V}^{\prime}=\left(\begin{array}{ccc}\frac{1}{4}\left(2 s_{11}+2 s_{12}+s_{44}\right) & \frac{1}{12}\left(2 s_{11}+10 s_{12}-s_{44}\right) & 0 \\ \frac{1}{12}\left(2 s_{11}+10 s_{12}-s_{44}\right) & \frac{1}{4}\left(2 s_{11}+2 s_{12}+s_{44}\right) & 0 \\ 0 & 0 & \frac{2}{3}\left(s_{11}-s_{12}+s_{44}\right)\end{array}\right)$,

$\mathbf{B}^{\prime}=\left(\begin{array}{ccc}\frac{1}{6}\left(2 s_{11}+4 s_{12}-s_{44}\right) & 0 & \frac{1}{3 \sqrt{2}}\left(-2 s_{11}+2 s_{12}+s_{44}\right) \\ \frac{1}{6}\left(2 s_{11}+4 s_{12}-s_{44}\right) & 0 & \frac{1}{3 \sqrt{2}}\left(2 s_{11}-2 s_{12}-s_{44}\right) \\ 0 & \frac{\sqrt{2}}{3}\left(2 s_{11}-2 s_{12}-s_{44}\right) & 0\end{array}\right)$,

$\mathbf{N}^{\prime}=\left(\begin{array}{ccc}\frac{1}{3}\left(s_{11}+2 s_{12}+s_{44}\right) & 0 & 0 \\ 0 & \frac{1}{3}\left(4 s_{11}-4 s_{12}+s_{44}\right) & 0 \\ 0 & 0 & \frac{1}{3}\left(4 s_{11}-4 s_{12}+s_{44}\right)\end{array}\right)$

out any disturbance. Then the spontaneous polarization of the film can be determined as

$P^{\mathrm{s}}=\sqrt{\frac{-\beta_{1}^{*}}{2 a_{11}^{*}}}$

and the dielectric constant as a function of the applied electric field can be determined from

$\chi_{33}\left(E^{0}\right)=\frac{\partial P_{3}}{\partial E^{0}}=\frac{1}{\beta_{1}^{*}+6 a_{11}^{*} P_{3}^{2}(\theta)}$ with the structures of $\mathbf{C}^{\prime}, \mathbf{D}^{\prime}$, and $\mathbf{M}^{\prime}$ similar to $\mathbf{V}^{\prime}, \mathbf{B}^{\prime}$, and $\mathbf{N}^{\prime}$, respectively. Substituting them into Eq. (12), the unknown stress and strain components for grain at orientation $\theta$ in the local coordinates are derived as

$$
\begin{aligned}
\sigma_{1}(\theta) & =\sigma_{2}(\theta) \\
& =\frac{6 \varepsilon^{0}-3 P^{2}(\theta)\left(2 Q_{11}+4 Q_{12}-Q_{44}\right)}{4 s_{11}+8 s_{12}+s_{44}}, \quad \sigma_{6}(\theta)=0
\end{aligned}
$$

and

$\varepsilon_{3}(\theta)=\frac{\varepsilon^{0}\left(4 s_{11}+8 s_{12}-2 s_{44}\right)-3 P^{2}(\theta)\left[2 Q_{44}\left(s_{11}+2 s_{12}\right)+\left(Q_{11}+2 Q_{12}\right) s_{44}\right]}{4 s_{11}+8 s_{12}+s_{44}}, \quad \varepsilon_{4}(\theta)=\varepsilon_{5}(\theta)=0$

from which the dielectric tunability $\phi$ can be obtained as

$\phi=1-\frac{\beta_{1}^{*}+6 a_{11}^{*}\left(P^{\mathrm{s}}\right)^{2}}{\beta_{1}^{*}+6 a_{11}^{*} P_{3}^{2}(\theta)}$

\subsection{Cubic and rhombohedral phase with [111] fiber texture}

For a ferroelectric thin film consisting of grains of rhombohedral phase, the spontaneous polarization in the local cubic coordinates is given by

$P_{1}=P_{2}=P_{3}=P(\theta)=0$ while the polarization can be solved from

$$
\begin{aligned}
& \sqrt{3} P(\theta) \beta_{1}+2 \sqrt{3} P^{3}(\theta)\left(a_{11}+a_{12}\right)-\frac{1}{\sqrt{3}} P(\theta) \\
& \quad \times\left(2 Q_{11}+4 Q_{12}-Q_{44}\right)\left[\sigma_{1}(\theta)+\sigma_{2}(\theta)\right]=E^{0}
\end{aligned}
$$

Here $b=a_{12} / 4$ is assumed for the rhombohedral phase. Substituting the stress components into the above polarization equation, we obtain

$$
\sqrt{3} P(\theta) \beta_{1}^{*}+2 \sqrt{3} P^{3}(\theta) a_{11}^{*}-E^{0}=0
$$


from which the cubic-rhombohedral phase transition temperature, the spontaneous polarization, and the dielectric constant can be determined as:

$T_{\mathrm{c}}=T_{0}+\frac{8 C \varepsilon_{0}\left(2 Q_{11}+4 Q_{12}-Q_{44}\right)}{4 s_{11}+8 s_{12}-2 s_{44}} \varepsilon^{0}$

$P^{\mathrm{s}}=\sqrt{3} P(\theta)=\sqrt{\frac{-3 \beta_{1}^{*}}{2 a_{11}^{*}}}$

$\chi_{33}\left(E^{0}\right)=\frac{\partial[\sqrt{3} P(\theta)]}{\partial E^{0}}=\frac{1}{\beta_{1}^{*}+6 a_{11}^{*} P^{2}(\theta)}$

where the nominal dielectric constants are defined as:

$\beta_{1}^{*}=\beta_{1}-\frac{4 \varepsilon^{0}\left(Q_{11}+2 Q_{12}-Q_{44}\right)}{4 s_{11}+8 s_{12}-s_{44}}$

$a_{11}^{*}=a_{11}+a_{12}+\frac{\left(Q_{11}+2 Q_{12}-Q_{44}\right)^{2}}{4 s_{11}+8 s_{12}-s_{44}}$

\section{Results and discussion}

We now apply the theory to study the dielectric behavior of $\mathrm{Ba}_{0.7} \mathrm{Sr}_{0.3} \mathrm{TiO}_{3}$ (BST 70/30) films of different symmetries and fiber textures. The constitutive moduli of the singlecrystalline BST 70/30 that we use in the following calculations are given in Table 1 [11].

\subsection{Cubic phase with [001] fiber texture}

We first consider a cubic phase polycrystalline BST film with [001] fiber texture, which is considered as a special case of tetragonal ferroelectric BST. The polarization, dielectric constant, and tunability of such a BST film as functions of the applied electric field subjected to different misfit strains at $150{ }^{\circ} \mathrm{C}$ are shown in Fig. 2. It is observed that both polarization and dielectric tunability increase with the applied electric field, with the dielectric tunability rapidly increasing until it reaches about $70 \%$ at around $50 \mathrm{MV} / \mathrm{m}$ when $\varepsilon^{0}=0$; the dielectric constant decreases with the applied electric field. This can be understood from Eqs. (19), (22) and (23) with positive $\beta_{1}^{*}$ and $a_{11}^{*}$. In particular, we notice that the increase of polarization decreases the dielectric constant while increasing the tunability. It is also observed that the compressive strain enhances the polarization, dielectric constant, and tunability, while tensile strain decreases them, consistent with experimental observations $[5,6]$. This is because the compressive strain decreases $\beta_{1}^{*}$ when $Q_{12}$ is negative, according to Eq. (18), leading to increased polarization, dielectric constant, and tunability, according to Eqs. (19), (22) and (23). As a result, the misfit strain can be applied to tune the dielectric properties of BST for microwave applications.
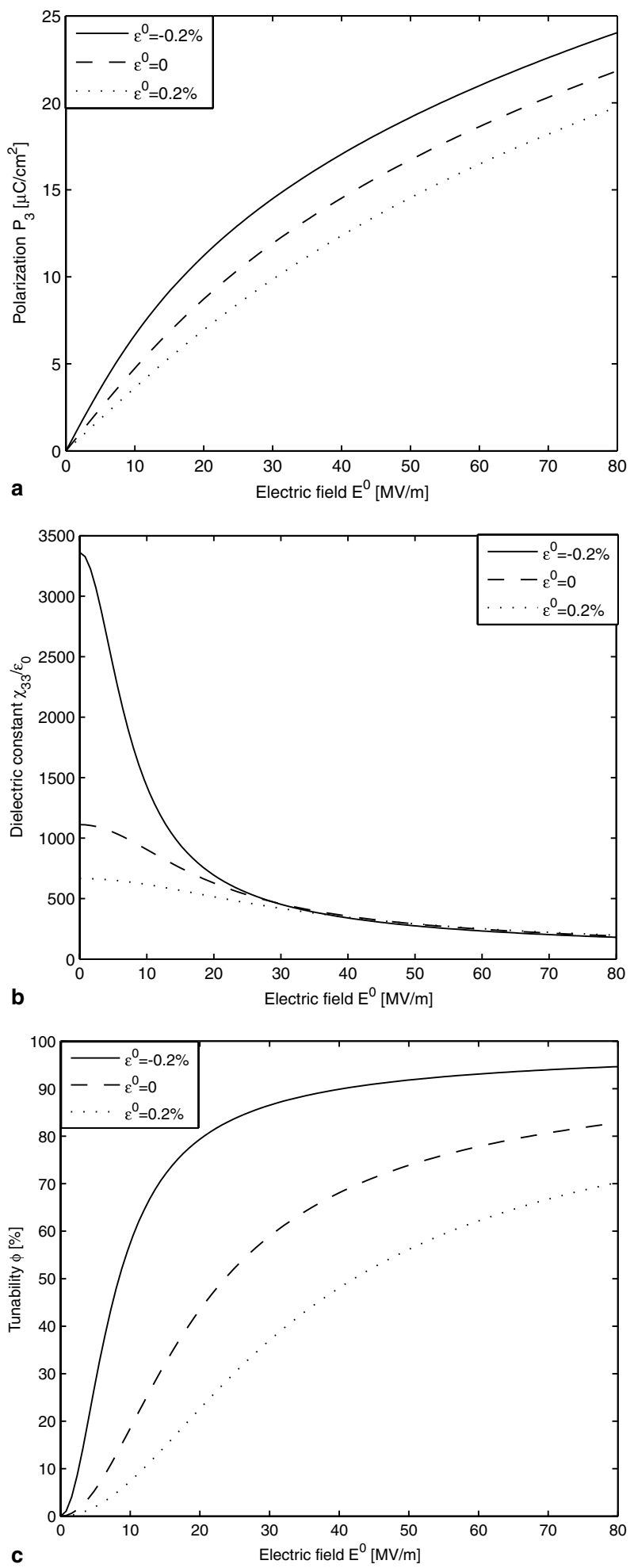

Fig. 2. Dielectric properties of cubic [001] BST as a function of applied field $E^{0}$ at different misfit strains: (a) polarization; (b) dielectric constant; (c) tunability.

Table 1

The constitutive moduli of single-crystalline BST 70/30 film [11]

\begin{tabular}{|c|c|c|c|c|c|c|c|c|c|}
\hline $\begin{array}{l}T_{0} \\
\left({ }^{\circ} \mathrm{C}\right)\end{array}$ & $\begin{array}{l}C \\
\left(10^{5}{ }^{\circ} \mathrm{C}\right)\end{array}$ & $\begin{array}{l}a_{11} \\
\left(10^{6} \mathrm{~m}^{5} / \mathrm{C}^{2} \mathrm{~F}\right)\end{array}$ & $\begin{array}{l}a_{12} \\
\left(10^{6} \mathrm{~m}^{5} / \mathrm{C}^{2} \mathrm{~F}\right)\end{array}$ & $\begin{array}{l}s_{11} \\
\left(10^{-12} \mathrm{~m}^{2} / \mathrm{N}\right)\end{array}$ & $\begin{array}{l}s_{12} \\
\left(10^{-12} \mathrm{~m}^{2} / \mathrm{N}\right)\end{array}$ & $\begin{array}{l}s_{44} \\
\left(10^{-12} \mathrm{~m}^{2} / \mathrm{N}\right)\end{array}$ & $\begin{array}{l}Q_{11} \\
\left(\mathrm{~m}^{4} / \mathrm{C}^{2}\right)\end{array}$ & $\begin{array}{l}Q_{12} \\
\left(\mathrm{~m}^{4} / \mathrm{C}^{2}\right)\end{array}$ & $\begin{array}{l}Q_{44} \\
\left(\mathrm{~m}^{4} / \mathrm{C}^{2}\right)\end{array}$ \\
\hline 34 & 0.65 & $5.04 T+378$ & 1442 & 5.92 & -1.92 & 6.7 & 0.1 & -0.034 & 0.029 \\
\hline
\end{tabular}


We also compare our calculated dielectric constants of BST $70 / 30$ film at $50{ }^{\circ} \mathrm{C}$ as a function of misfit strain with experiment data for highly textured BST 70/30 films grown on Si substrates [15,11], as shown in Fig. 3. Good agreement is observed especially when the misfit strain is small. It is noted that the misfit strain used here is calculated from film thickness, as originally proposed by Ban and Alpay [11]. In addition, we compared the variation of the polarization with the electric field for the $80 \mathrm{~nm} \mathrm{BST} 70 / 30$ film at $20{ }^{\circ} \mathrm{C}$ [15], and again good agreement is observed as shown in Fig. 4, especially when the electric field is modest. At higher electric field, a higher order dielectric constant is probably necessary to model the dielectric behavior more accurately.

\subsection{Cubic phase with [1111] fiber texture}

Next we consider the polarization, dielectric constant, and dielectric tunability of cubic phase BST film with

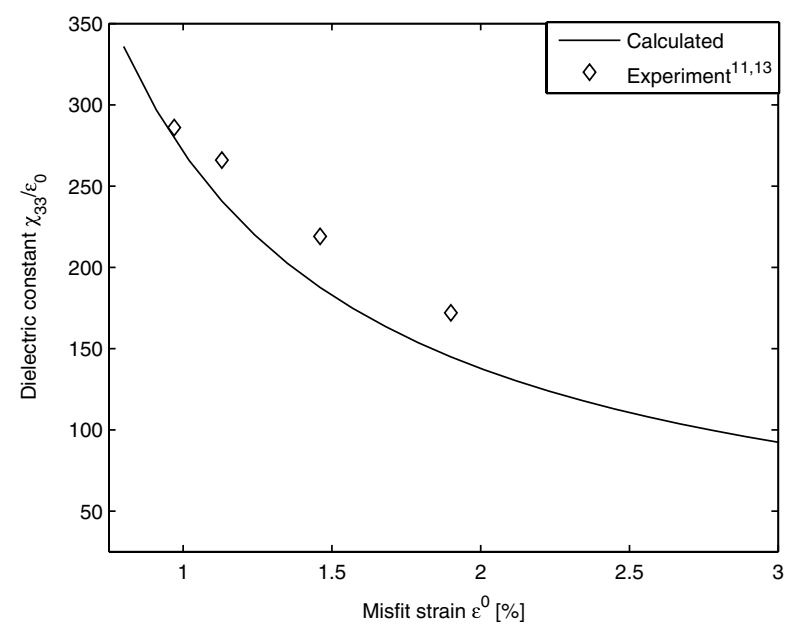

Fig. 3. Comparison between the theoretical calculations of dielectric constant $\chi_{33} / \varepsilon_{0}$ of BST $70 / 30$ film with reported experimental results.

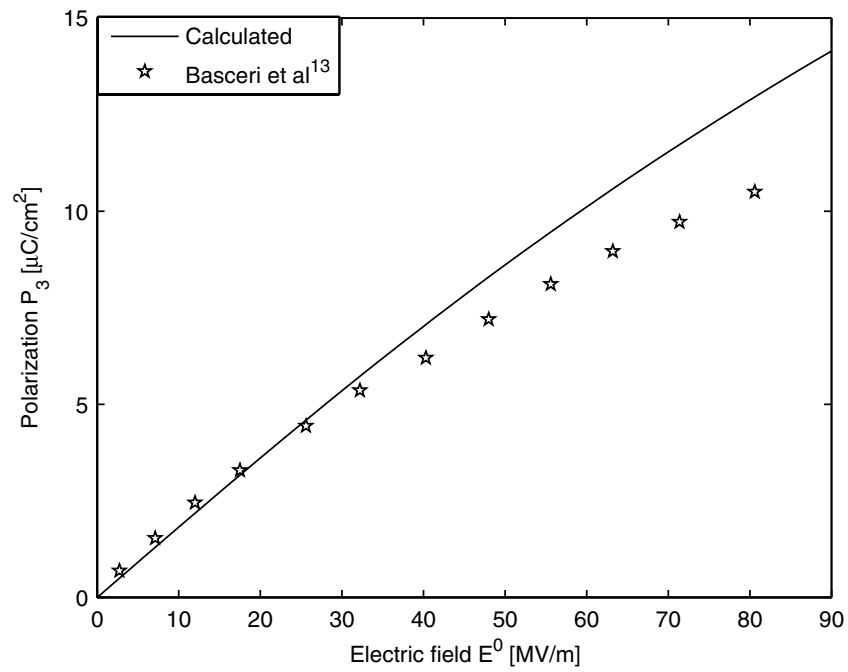

Fig. 4. Polarization vs. electric field, calculated from the measured smallsignal $C-V$ data for the $80 \mathrm{~nm}$ BST 70/30 film and our calculated results at $200^{\circ} \mathrm{C}$.
[111] fiber texture at a temperature $T=150^{\circ} \mathrm{C}$, which is regarded as a special case of the rhombohedral phase. From Fig. 5, it is observed that both the polarization and
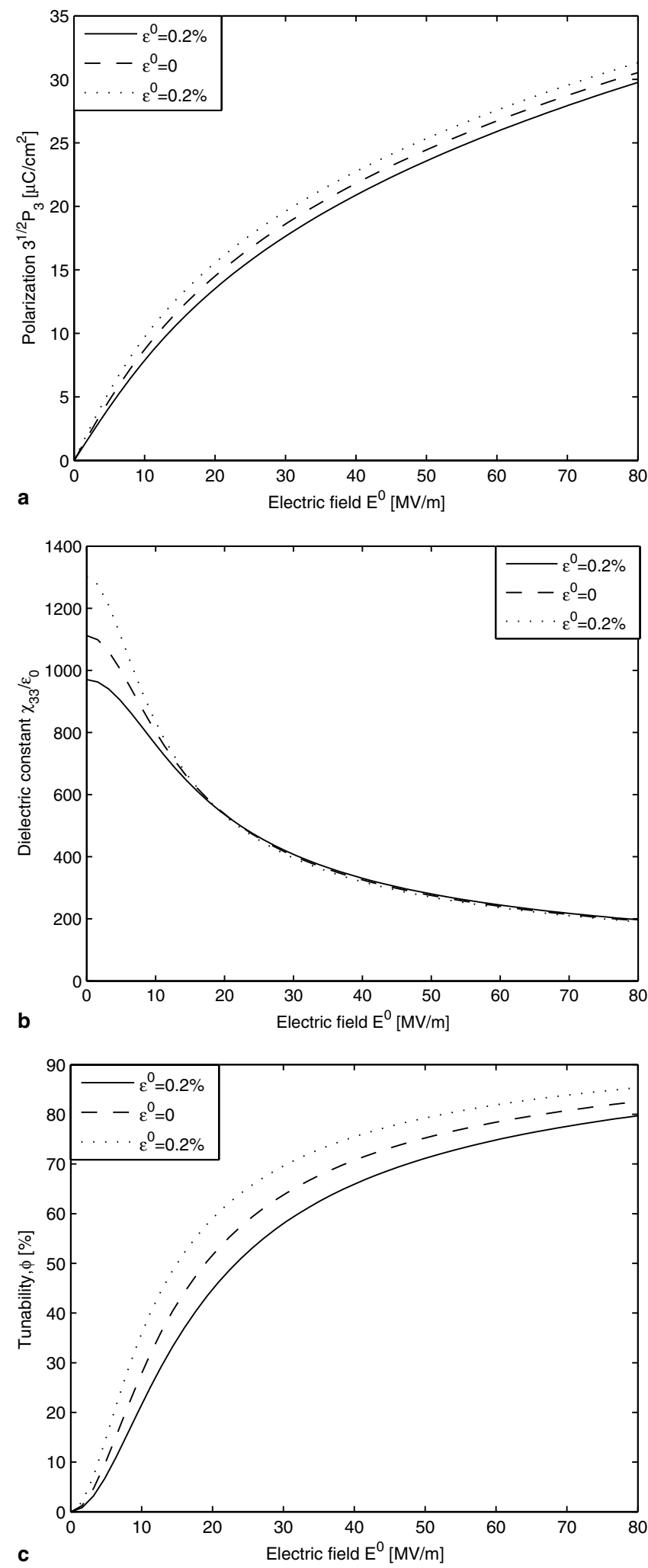

Fig. 5. Dielectric properties of cubic [1 111 ] fiber-textured BST film as a function of applied field $E^{0}$ at different misfit strain: (a) polarization; (b) dielectric constant; (c) tunability. 
tunability increase while the dielectric constant decreases with the applied electric field, similar to the results for [0 01 1] fiber-textured cubic BST. However, it is noted that, unlike cubic BST with [001] fiber texture, the tensile strain enhances the polarization, dielectric constant, and tunability, while compressive strain decreases them. This is because $Q_{11}+2 Q_{12}-Q_{44}$ in the expression for $\beta_{1}^{*}$ of Eq. (30) is positive, opposite to $Q_{12}$ of [001]-textured film. In other words, under an electric field applied along the normal direction of the film, the [001]-textured film would contract in-plane, while the [111]-textured one would expand instead, and this difference leads to the different responses to misfit strain. In addition, we notice the [1 111 -textured film is less sensitive to the variation of the misfit strain than the $[001]$-textured one.

\subsection{Tetragonal phase with [001] fiber texture}

We now consider a ferroelectric BST film in tetragonal phase with [001] fiber texture at a temperature $T=0{ }^{\circ} \mathrm{C}$, with hysteresis, dielectric constant, and tunability as functions of the applied field at different misfit strains shown in Fig. 6. It is observed that compressive misfit strain increases both the spontaneous polarization and coercive field, while at the same time decreases the dielectric peak near the polarization switching. This is because, for the tetragonal phase, the nominal dielectric $\beta_{1}^{*}$ is negative, and its magnitude increases with the compressive strain because of the negative sign of $Q_{12}$ in Eq. (18), leading to enhanced polarization, and thus enhanced coercivity since higher polarization makes it more difficult to switch. Meanwhile, a higher polarization and a larger negative $\beta_{1}^{*}$ make the dielectric constant smaller, as seen from Eq. (22). It is worth noting that the misfit strain can also change significantly the characteristics of the cubictetragonal phase transition. To illustrate this, we plot the variation of the polarization and dielectric constant of [001]-textured film as a function of misfit strain at room temperature with no applied electric field, as shown in Fig. 7. It is observed that the spontaneous polarization decreases with the decreasing compressive strain, and at a critical tensile strain $\varepsilon^{0}=0.023 \%$, the spontaneous polarization becomes zero, and the corresponding dielectric constant shows a peak, indicating that there is a tetragonal-cubic phase transition and a tensile misfit strain disfavoring the ferroelectric tetragonal phase. This can also be understood from Eq. (20), which suggests that a compressive strain increases the phase transition temperature for the [001]-textured film while a tensile strain decreases it.

\subsection{Rhombohedral phase}

Finally, we consider a ferroelectric BST film in rhombohedral phase with [111] fiber texture at a temperature $T=-50^{\circ} \mathrm{C}$, with hysteresis, dielectric constant, and tunability as functions of the applied field at different misfit
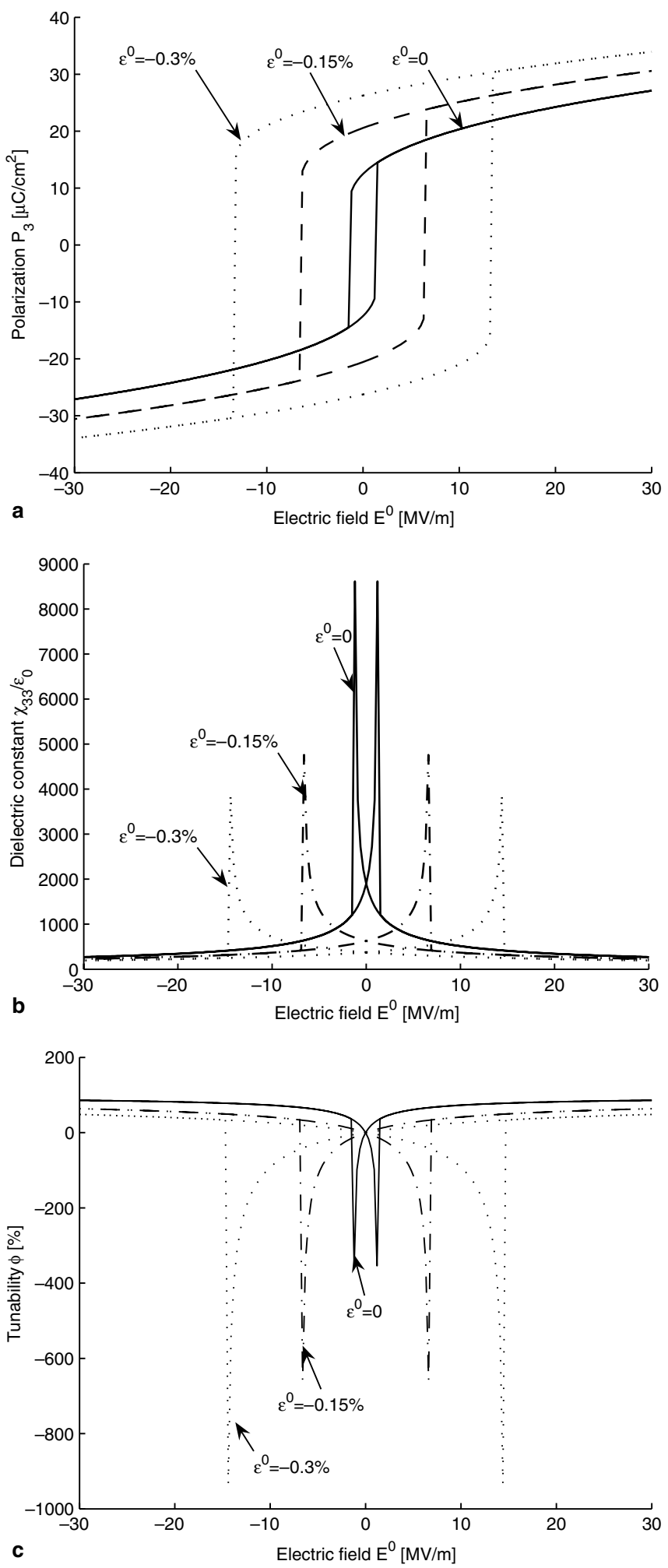

Fig. 6. Dielectric properties of tetragonal BST as a function of applied field $E^{0}$ at different misfit strains: (a) hysteresis loop of polarization; (b) butterfly loop of dielectric constant; (c) tunability.

strains shown in Fig. 8. It is observed that, in contrast to the results for the tetragonal phase, the compressive strain decrease the spontaneous polarization and coercive field while it increases the peak value of the dielectric constant 

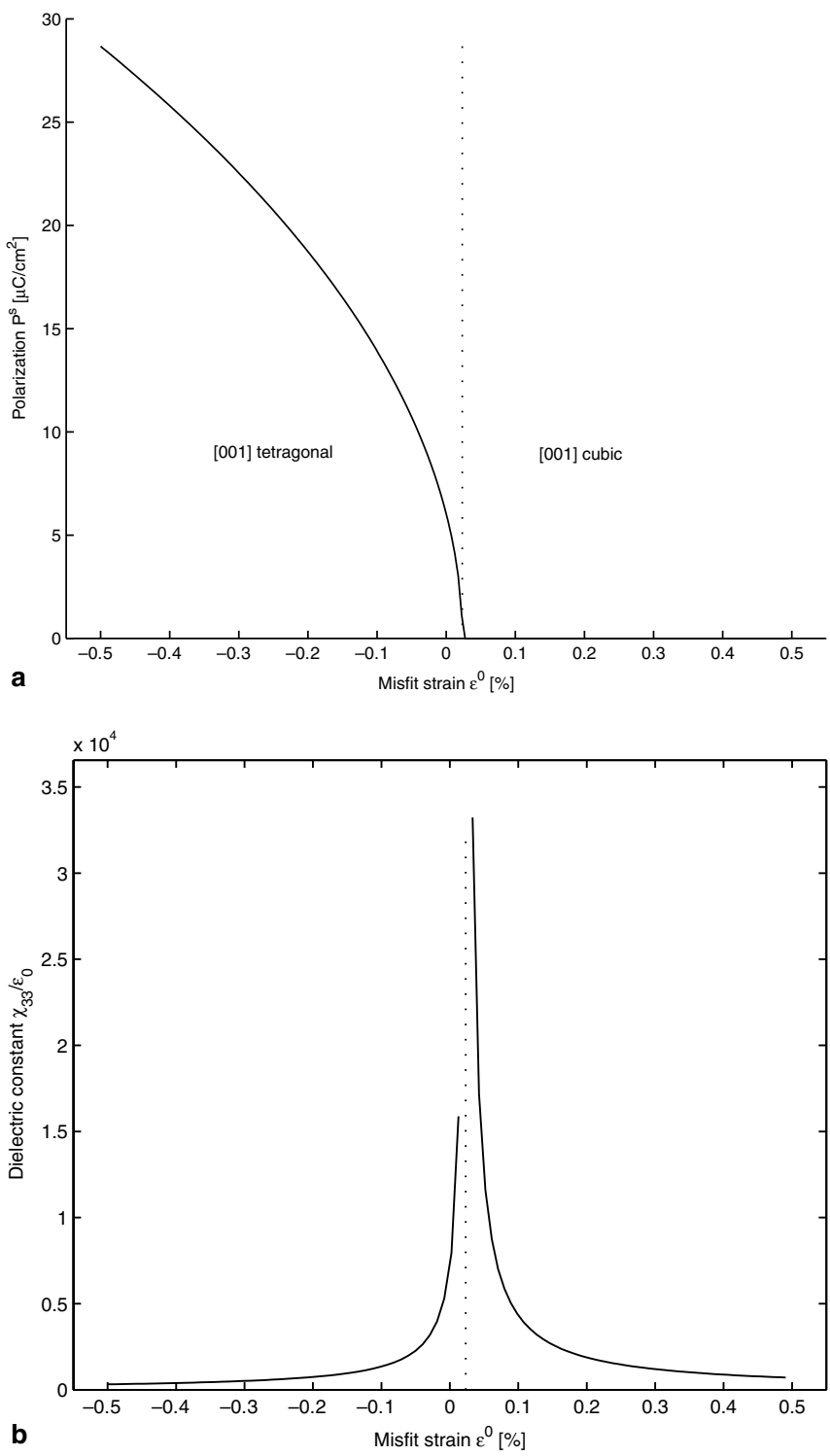

Fig. 7. Polarization (a) and dielectric constant (b) as a function of misfit strain for [001] fiber-textured BST 70/30 film at room temperature without an applied electric field.

near polarization reversal. This is, again, due to the positive value of $Q_{11}+2 Q_{12}-Q_{44}$ in the expression for $\beta_{1}^{*}$ of Eq. (30). Moreover, we notice that the [111] -textured rhombohedral phase is much less sensitive to the misfit strain than the [001]-textured tetragonal phase, which is similar to what we observed for the cubic phase with [1 111$]$ and [00 1] fiber textures. To study the effect of misfit strain on phase transition characteristics, we plot the variation of the polarization and dielectric constant of the [1 111 ] -textured film as a function of misfit strain at room temperature with no applied electric field, as shown in Fig. 9. It is observed that in contrast to [001]-textured tetragonal films, the spontaneous polarization increases with the tensile strain. At a critical strain $\left(\varepsilon^{0}=-0.11 \%\right)$ the spontaneous polarization becomes zero, and the corre-
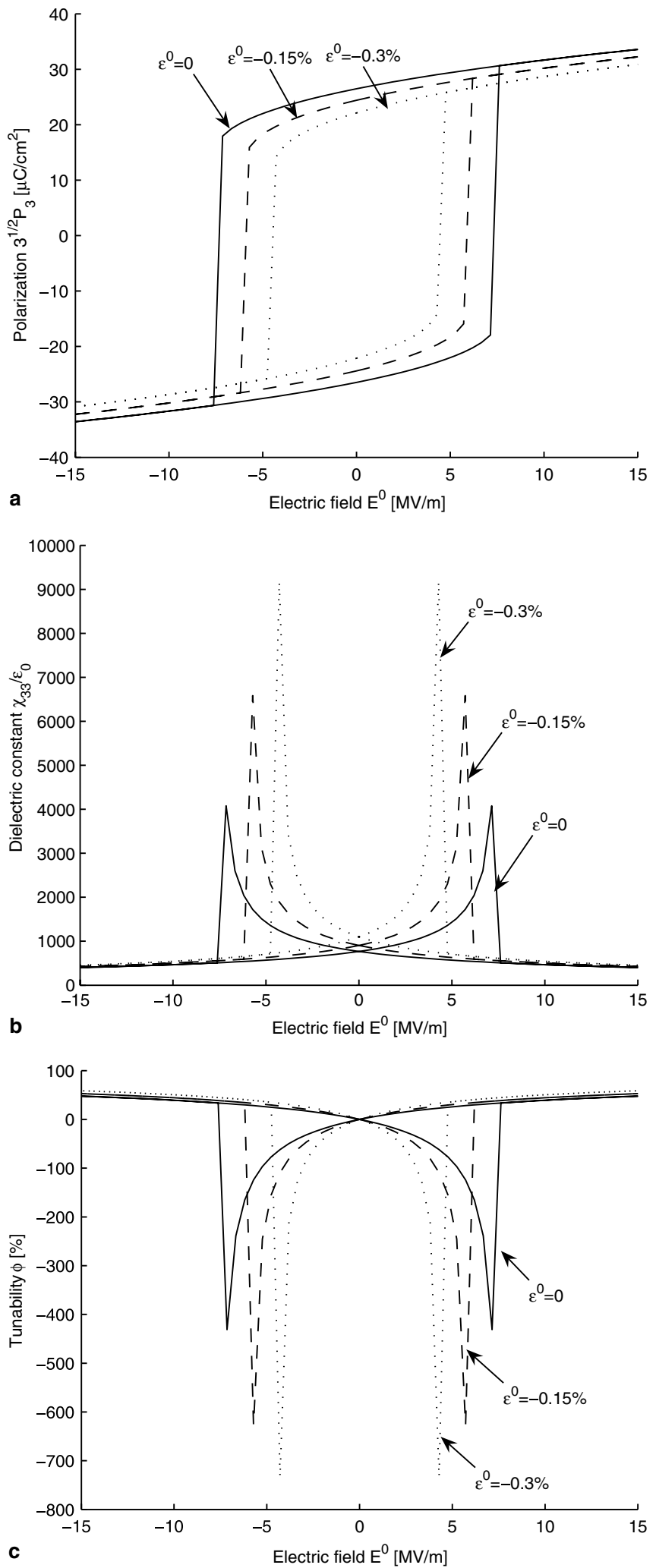

Fig. 8. Dielectric properties of rhombohedral BST as a function of applied field $E^{0}$ at different misfit strain: (a) hysteresis loop of polarization; (b) butterfly loop of dielectric constant; (c) tunability.

sponding dielectric constant shows a peak, indicating that there is a rhombohedral-cubic phase transition and a compressive strain disfavoring the ferroelectric rhombohedral 

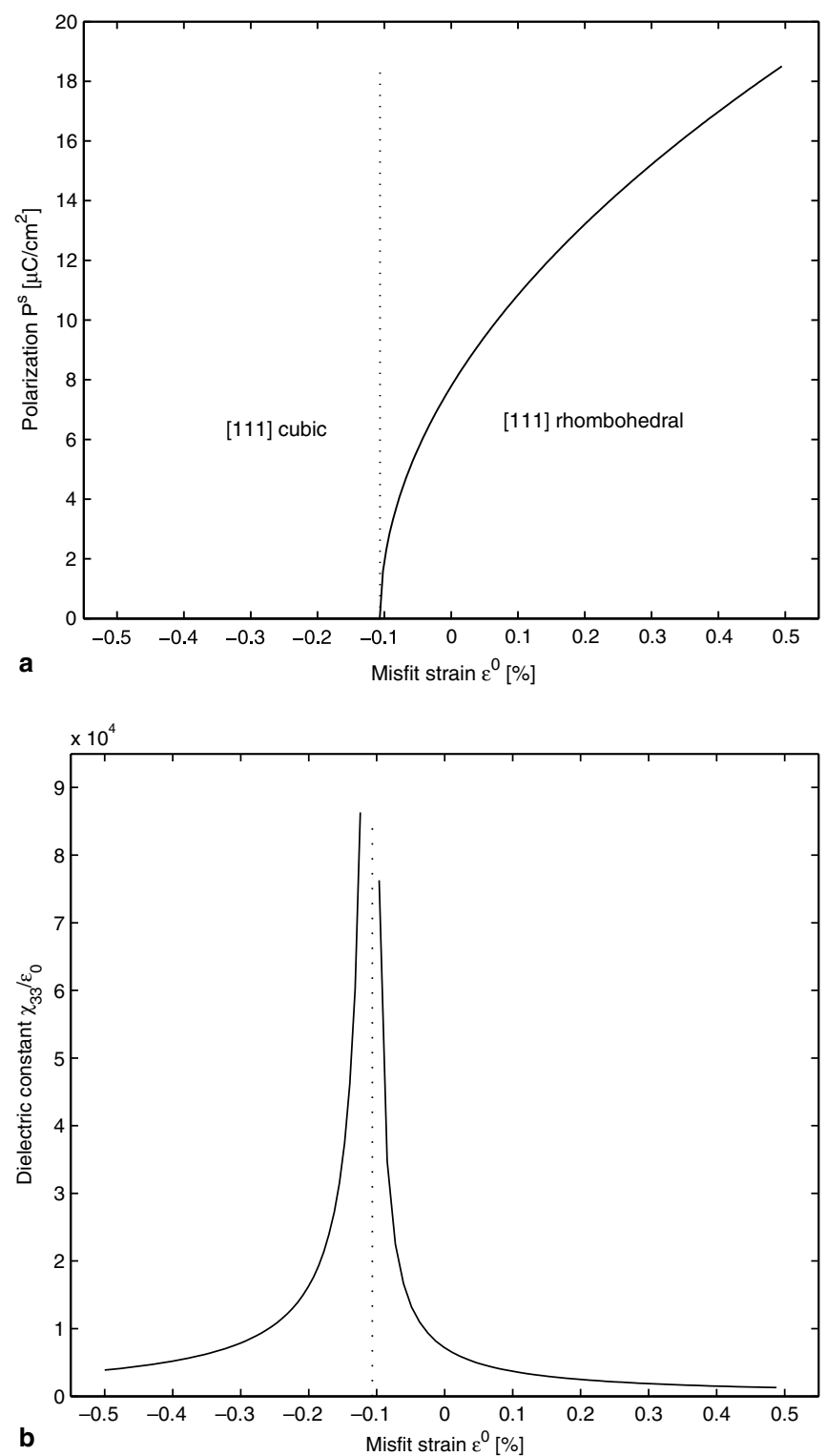

Fig. 9. Polarization (a) and dielectric constant (b) as a function of misfit strain for [111] fiber-textured BST $70 / 30$ film at room temperature without an applied field.

phase. The difference between [0011]- and [1 111$]$-textured films can again be explained by the different signs in the expression for $\beta_{1}^{*}$.

\section{Summary}

We have combined Landau-Ginzburg-Devonshire phenomenological theory with the micromechanics theory to study the dielectric behavior of ferroelectric polycrystalline films with fiber textures, where the effects of crys- tallographic symmetry, texture, and misfit strain on the spontaneous polarization, coercivity, dielectric constant, and dielectric tunability have been considered, and the strain-induced phase transition has also been analyzed. The calculated dielectric properties are in good agreement with the experimental data reported in the literature.

\section{Acknowledgements}

This research was sponsored by the US Army Research Laboratory under the RMAC-RTP Cooperative Agreement No. W911NF-04-2-0011 and the National Science Foundation under CMS-0415261. Most of the work was carried out while Q.D. and J.Y.L. were at the University of Nebraska-Lincoln.

\section{References}

[1] Scott JF, Ross FM. Ferroelectrics 1997;201:43.

[2] Damjanovic D. Rep Prog Phys 1998;61:1267.

[3] Shaw TM, Trolier-McKinstry S, McIntyre PC. Annu Rev Mater Sci 2000;30:263.

[4] Bhattacharya K, James RD. J Mech Phys Solids 1999;47:531.

[5] Canedy CL, Li H, Alpay SP, Salamanca-Riba L, Roytburd AL, Ramesh R. Appl Phys Lett 2000;77:1695.

[6] Li H, Roytburd AL, Alpay Tran SP, Tran TD, Salamanca-Riba L, Ramesh R. Appl Phys Lett 2001;78:2354.

[7] Babbitt RW, Koscica TE, Drach WC. Microwave J 1992;35:63.

[8] Cole MW, Geyer RG. Mech Mater 2004;36:1017.

[9] Rossetti GA, Cross LE, Kushida K. Appl Phys Lett 1991;59:2524.

[10] Pertsev NA, Zembilgotov AG, Tagantsev AK. Phys Rev Lett 1998;80:1988.

[11] Ban ZG, Alpay SP. J Appl Phys 2002;91:9288.

[12] Li JY, Rogan RC, Üstündag E, Bhattacharya K. Nature Mater 2005;4:776.

[13] Li JY, Dunn ML, Ledbetter H. J Appl Phys 1999;86:4626.

[14] Zhou YC, Liu J, Li JY. Appl Phys Lett 2005;86:262909.

[15] Basceri C, Streiffer SK, Kingon AI, Waser R. J Appl Phys 1997:82:2497.

[16] Norga GJ, Jin S, Fe L, Wouters DJ, Bender H, Maes HE. J Mater Res $2001 ; 16: 828$.

[17] Haeni H, Irvin P, Chang W, Uecker R, Reiche P, Li YL, et al. Nature 2004;430:758.

[18] Park BH, Peterson EJ, Jia QX, Lee J, Zeng X, Si W, et al. Appl Phys Lett 2001;78:533.

[19] Catalan G, Noheda B, McAneney J, Sinnamon LJ, Gregg JM. Phys Rev B 2005;72:020102.

[20] Wang J, Zhang T. Appl Phys Lett 2005;86:192905.

[21] Morito K, Suzuki T. J Appl Phys 2005;97:104107.

[22] DeSimone A. Arch Rat Mech Anal 1993;125:99.

[23] Shu YC, Bhattacharya K. Philos Mag B 2001;81:2021.

[24] Nye JF. Physical properties of crystals. Oxford: Oxford University Press; 1957.

[25] Liu D, Li JY. Appl Phys Lett 2003;83:1193.

[26] Liu D, Li JY. Appl Phys Lett 2004;84:3930.

[27] Li JY, Liu D. J Mech Phys Solids 2004;52:1719. 